\title{
Phaseless Single-Step Microwave Imaging Technique for Biomedical Applications
}

\author{
Sandra COSTANZO, Giuseppe LOPEZ \\ DIMES, University of Calabria, Via P. Bucci 42C, 87036 Rende (CS), Italy \\ costanzo@dimes.unical.it
}

Submitted July 15, 2019 / Accepted July 15, 2019

\begin{abstract}
In the present work, an improved phaseless approach to microwave imaging is presented. Starting from the Contrast Source formulation of the scattering problem, a single-step procedure with no intermediate phase-retrieval process is described. The reconstruction capabilities of the proposed phaseless inverse method are numerically validated by firstly considering simple dielectric targets. Then, a slice breast model with the inclusion of a cancerous portion is analyzed. The identification of different types of breast tissue is successfully achieved, thus confirming the validity and potentialities of the proposed phaseless technique in the framework of biomedical imaging.
\end{abstract}

\section{Keywords}

Electromagnetic (EM) inverse scattering problem, Phaseless Contrast-Source Inversion method (P-CSI), breast imaging

\section{Introduction}

Microwave imaging (MWI) techniques are gaining increasing interest in medical diagnostics, due to the adoption of non-ionizing radiation as well as the provided low Specific Absorption Rate (SAR), further combined with a cheaper, non-invasive and compact imaging setup. However, the limited penetration depth and the relatively low resolution give a strong limitation in the large-scale deployment of microwave tomography (MWT). The potentialities of MWI to provide information about the health status of inaccessible tissues, with a better dielectric representation of biological materials, suggest to combine this technique with the widely used X-ray and Magnetic Resonance Imaging (MRI). Furthermore, MWT is revealed to be less prone to false-positive and false-negative rates, as compared to conventional diagnostics techniques [1]. According to a first preliminary investigation presented in [2], a Phaseless Contrast Source Inversion Method (P-CSI) is successfully implemented in the present work for breast tissue reconstruction. In particular, a Contrast Source (CS) formulation is adopted [3], [4], and the inverse scattering problem is solved with no linearization procedure, by converting it into an iterative optimization problem, where the two unknowns, namely the contrast source and the dielectric contrast, are alternatively updated according to a conjugate gradient scheme. The inversion procedure is performed by exploiting the amplitude-only data of the measured total field, locally defined as the sum of the incident and the scattered fields, the former obtained as a base-line measurement in the absence of the Object Under Test (OUT), and the latter due to the interaction of the incident field with the OUT. The full-data information of the incident field (which can be easily extracted from simulations) is also required for the reconstruction process.

\section{Scattering Problem Formulation}

Let us consider a two-dimensional tomography problem, aiming at localize a generic OUT and retrieve its dielectric properties, hereby denoted as B. A TM-polarized incident wave is considered, and cylindrical targets are analyzed as well. A magnetic permittivity equal to that of free-space is assumed, to match with the non-magnetic property of a biomedical scenario. Measurement points are equally distributed on the acquisition curve $\mathbb{S}$, as shown in the general scheme of Fig. 1.

It describes a multi-static and multi-view setup, where the transmitter location is alternatively changed, with $N_{\text {TX }}$ angles of incidence, resulting in a number $N_{\mathrm{RX}} \times N_{\mathrm{TX}}$ of

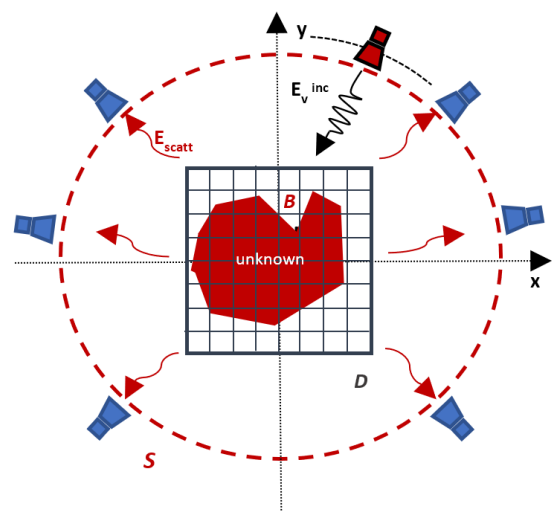

Fig. 1. Configuration of acquisition setup. 
measurements. The imaging domain, also named Domain of Interest (DOI) and hereby indicated as $\mathbb{D}$, fully contains the unknown OUT.

According to the theory [5], [6], the scattering problem can be analyzed by considering the following equations, known as Electrical Field Integral Equations (EFIEs):

$$
\begin{gathered}
E^{\mathrm{t}}(r)=E^{\mathrm{i}}(r)+k_{\mathrm{b}}{ }^{2} \int_{\mathbb{D}} G\left(r, r^{\prime}\right) \chi\left(r^{\prime}\right) E^{t}\left(r^{\prime}\right) \mathrm{d} r^{\prime}, r \in \mathbb{S}, \\
E^{\mathrm{s}}(r)=k_{\mathrm{b}}{ }^{2} \int_{\mathbb{D}} G\left(r, r^{\prime}\right) \chi\left(r^{\prime}\right) E^{t}\left(r^{\prime}\right) \mathrm{d} r^{\prime}, r \in \mathbb{D} .
\end{gathered}
$$

In particular, equation (1), indicated as data equation, relates the measured scattered field along the acquisition domain $\mathbb{S}$ with the dielectric contrast, conventionally expressed in a normalization form with respect to the background permittivity and indicated as $\chi$ :

$$
\chi(r)=\frac{k^{2}}{k_{\mathrm{b}}^{2}}-1
$$

$k_{\mathrm{b}}$ indicating the background wavenumber.

Equation (2), known as state equation, relates the total field, evaluated inside the DOI, with the dielectric properties inside the domain $\mathbb{D}$, wherein the object $\mathbb{B}$ is assumed to be delimited.

Let us assume $V$ different positions given by a TM source; for a fixed position, hereby indicated by $v$, the measured field is sampled along the acquisition curve by changing the location of the receiving antenna. Thus, series of measurement campaigns are performed. According to the aforementioned notation, the EFIEs can be reformulated in a compact form, by introducing the contrast source term, namely $\omega(r)=\chi(r) E^{\mathrm{t}}(r)$. We can write then:

$$
\begin{gathered}
E_{v}^{\mathrm{t}}(r)=E^{\mathrm{i}}(r)+\mathrm{G}_{\mathrm{S}} \omega_{v}(r), r \in \mathbb{S}, \\
\omega_{v}(r)=\chi(r)\left[E_{v}^{\mathrm{i}}(r)+\mathrm{G}_{\mathrm{D}} \omega_{v}(r)\right], r \in \mathbb{D}
\end{gathered}
$$

where $G$ denotes the same radiation operator for both equations, with the respective limitation in the range of $r$ and easily expressed in terms of Hankel function of the zero-th order and second kind.

In the Contrast Source Imaging (CSI) method, the solution of the tomographic problem requires the definition of a quadratic cost functional to be minimized. It is obtained as the combination of two sub-functionals, related to the misfit between measured and computed data, according to (4), (5).

From the above considerations, it results:

$$
\mathrm{F}\left(\omega_{v}, \chi\right):=\mathrm{F}_{\mathrm{S}}\left(\omega_{v}\right)+\mathrm{F}_{\mathrm{D}}\left(\omega_{v}, \chi\right)
$$

where $\mathrm{F}_{\mathrm{S}}$ and $\mathrm{F}_{\mathrm{D}}$ are denoted as data function and state function, respectively, and they can be expressed as:

$$
\mathrm{F}_{\mathrm{S}}\left(\omega_{v}\right)=\alpha_{\mathrm{S}} \sum_{v}\left\|\left.|| f_{v}\right|^{2}-\left|E_{v}^{\mathrm{i}}+\mathrm{G}_{\mathrm{S}} \omega_{v}\right|^{2}\right\|_{\mathbb{S}}^{2},
$$

$$
\mathrm{F}_{\mathrm{D}}\left(\omega_{v}\right)=\alpha_{\mathrm{D}} \sum_{v}\left\|\chi\left(E_{v}^{\mathrm{i}}+\mathrm{G}_{\mathrm{D}} \omega_{v}\right)-\omega_{v}\right\|_{\mathbb{D}}^{2}
$$

where $\alpha_{\text {S-D }}$ are proper normalization factors, while $f_{v}$ indicates the measured total field along the acquisition curve.

As it can be easily observed from (7), (8), amplitudeonly data for the total field are exploited, while the incident field is assumed in both amplitude and phase. A suitable incident field inside the DOI is obtained by looking for a computed matching incident field along the acquisition curve first [7], [8]. The latter is obtained as a linear combination of a limited number $N$ of Hankel functions of the second kind, so that the following relation holds true:

$$
E^{\mathrm{i}}(\rho, \theta) \cong-\frac{\mathrm{j}}{4} \sum_{v=-N}^{N} c_{\nu} \mathrm{H}_{v}{ }^{(2)}(k \rho) \exp (\mathrm{j} v \theta)
$$

where $(\rho, \vartheta)$ stands for polar coordinates.

In the second step, coefficients $c_{\mathrm{v}}$ are re-used for the determination of the incident field inside $\mathbb{D}$ [9].

The reconstruction procedure requires the CS initialization. The choice for this initial value into the optimization algorithm results to be a key point for the imaging process, since the convergence of the local optimization problem can be strongly influenced by the above initial guess.

According to the original work [3] introducing the CSI method, the use of the back-propagation (BP) approach for the initial estimate for the CS requires the fulldata availability of the total field. Consequently, further techniques have been proposed in literature with the aim to exploit the BP method in a phaseless approach, by considering an arbitrary phase for the total field [10], [11]. However, due to the lack of reason behind the phase selection, an alternative solution for the CS initialization is considered in the present work. This is obtained by applying the steepest descent method to the data function $F_{S}$, limited to the first step. In accordance with the aforementioned approach, it consequently results:

$$
\omega_{v, 0}=-2 \beta \alpha_{\mathrm{S}} \mathrm{G}_{\mathrm{S}}^{*}\left[E_{v}^{\mathrm{i}}\left(\left|f_{v}\right|^{2}-\left|E_{v}^{\mathrm{i}}\right|^{2}\right)\right]
$$

where the symbol * denotes the adjoint operator, while parameter $\beta$ indicates the step size. According to (10), all available data can be fully exploited into the initialization process; furthermore, no a priori information about the contrast inside $\mathbb{D}$ is required, as compared to the approach outlined in [12], where an approximate knowledge of the average contrast over the DOI is necessary.

\section{Numerical Validations on Dielectric Targets Reconstruction}

A first numerical validation of the proposed method is performed by using available measured data from Fresnel 


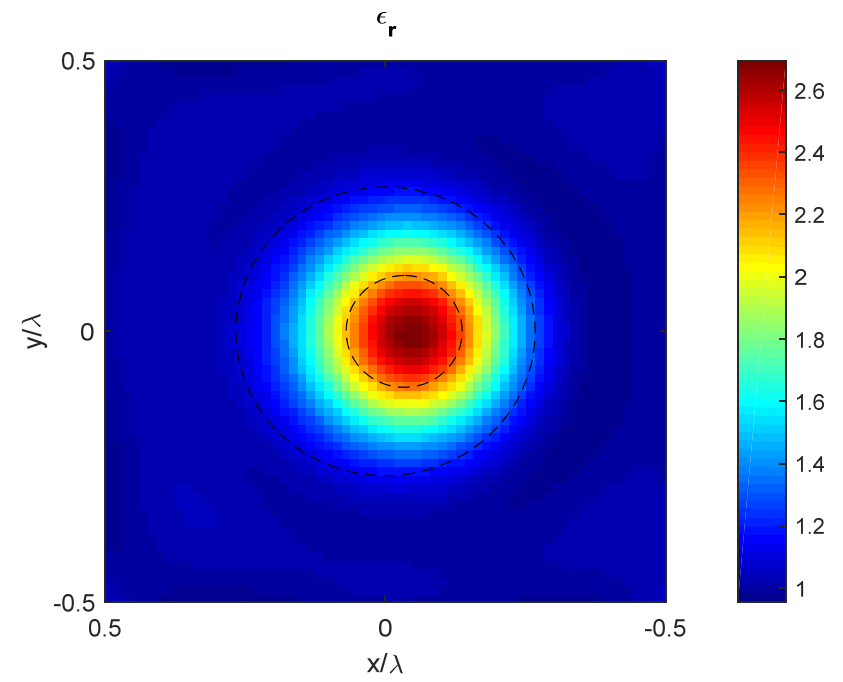

Fig. 2. Permittivity map for the target FoamDielInt in [13].

\begin{tabular}{|c|c|c|c|c|}
\hline FoamDielInt & $\boldsymbol{\varepsilon}_{\mathbf{r}}$ & Min & Mean & Max \\
\hline Air & 1 & 0.97 & 1.00 & 1.08 \\
\hline Foam & $1.45 \pm 0.15$ & 0.98 & 1.29 & 1.86 \\
\hline Plastic & $3 \pm 0.3$ & 2.27 & 2.43 & 2.78 \\
\hline
\end{tabular}

Tab. 1. Reconstructed permittivity values @ $f=2 \mathrm{GHz}$, with a maximum number of iterations equal to 150 .

Institute. They are relative to cylindrical dielectric targets combined in different fashions, thus resulting in homogeneous as well as inhomogeneous targets [13], [14].

The algorithm is tested for a fixed operating frequency, while the reconstruction process is performed until a convergence criterion on the cost functional $\mathrm{F}\left(\omega_{v}, \chi\right)$ is met or, alternatively, when a fixed maximum number of iterations is exceeded. An example of dielectric reconstruction is shown in Fig. 2, whereas the retrieved permittivity values are indicated in Tab. 1 .

\section{Breast Tissue Modeling and Imag- ing Results}

In order to confirm the reconstruction capabilities of the proposed method for breast imaging applications, a slice breast model is implemented on COMSOL Multiphysics ${ }^{\circledR}$ platform [15]. A simplified 3-tissue breast model is considered, in which a malignant portion with a $5 \mathrm{~mm}$ radius is included into the adipose tissue; the respective dielectric properties are determined similarly to [16] for a $2 \mathrm{GHz}$ operating frequency and are listed in Tab. 2, while a matching medium with $\varepsilon_{\mathrm{rb}}=12$ is assumed.

The detection of the tumor location comes from the permittivity contrast due to the different water content of the malignant tissues compared to the fat, combined with

\begin{tabular}{|c|c|c|}
\hline Tissue & $\boldsymbol{\varepsilon}_{\mathrm{r}}$ & $\boldsymbol{\sigma}$ \\
\hline Fat & 5 & 0.05 \\
\hline Fibroglandular tissue & 43 & 0.7 \\
\hline Tumor & 35 & 0.7 \\
\hline
\end{tabular}

Tab. 2. EM properties of breast tissues.

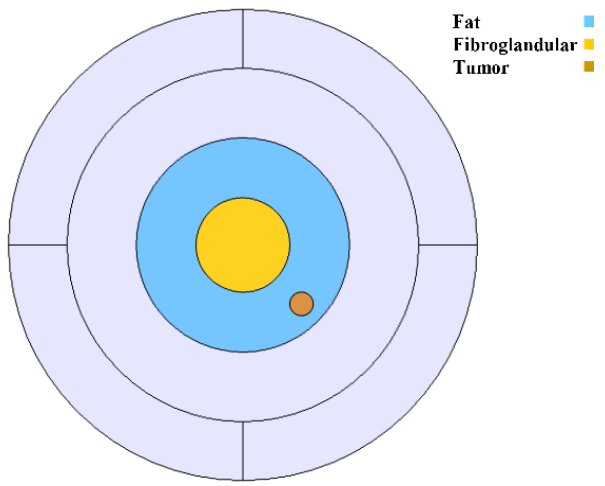

Fig. 3. Slice breast model on COMSOL platform.

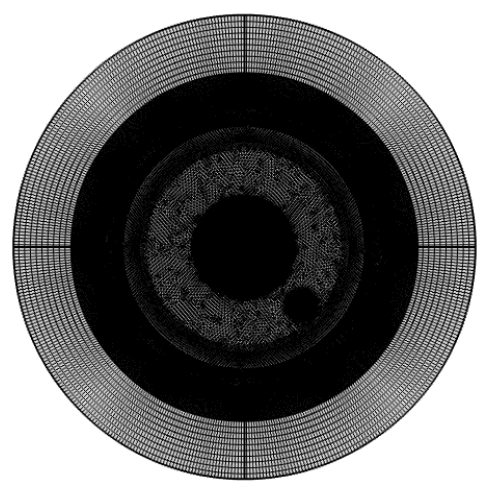

Fig. 4. Computational mesh and PML on COMSOL platform.

the higher conductivity of the cancerous portions. The breast for the $2 \mathrm{D}$ modeling is emulated with a cylinder having a $45.5 \mathrm{~mm}$ radius, while the fibroglandular portion has a radius equal to $20 \mathrm{~mm}$. The computational domain filled by the background medium needs to be truncated with the definition of a perfectly matched layer (PML) as boundary condition (Fig. 3). The correspondent computational mesh is shown in Fig. 4. Point sources are implemented for the TM field generation, and thus a series of line current out-of-plane are considered on COMSOL platform, in order to speed-up the forward computation needed for the generation of the synthetic data.

As shown in Fig. 5, a quantitative reconstruction of the contrast function $\chi$ and, consequently, of the relative permittivity is obtained for the lower-contrast regions. In particular, the cancerous region is clearly localized in the right corner.

The current state of the algorithm allows to retrieve a full-dielectric map in the case of relatively low-contrast scenario. In the case of high permittivity contrast, a qualitative reconstruction is guaranteed; additional developments are currently performed to optimize the performances.

\section{Conclusion}

The potentialities of a phaseless microwave tomography approach for biomedical imaging applications has been described and discussed in this work. Firstly, the numerical 


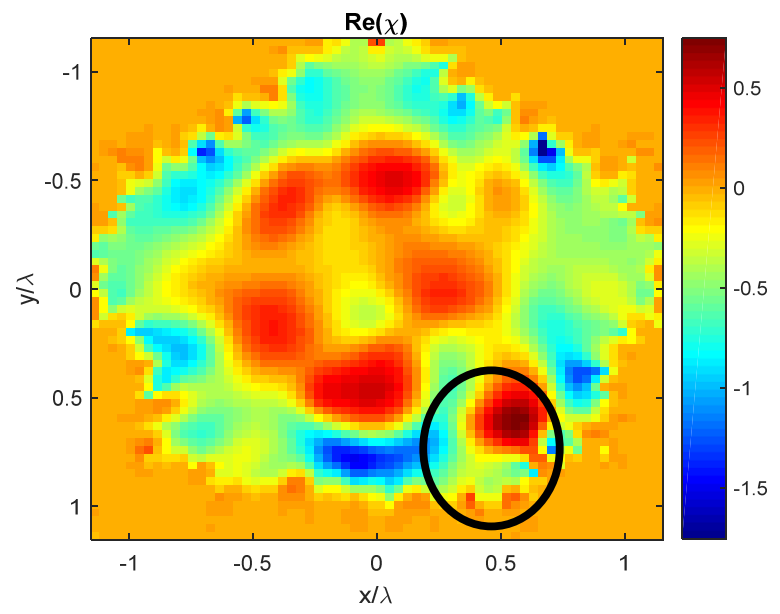

(a)

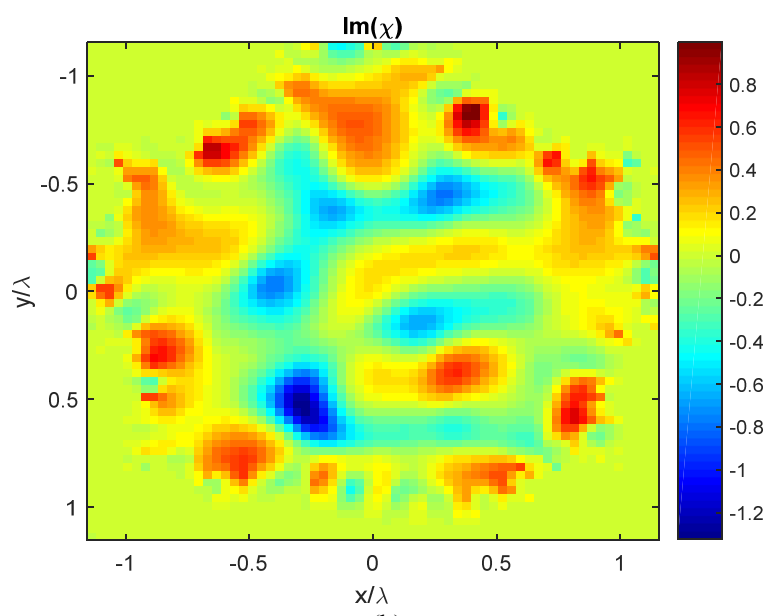

(b)

Fig. 5. Real (a) and imaginary (b) part of the dielectric contrast $\chi$ for the slice breast model.

validation of the proposed method has been performed by the reconstruction of a series of dielectric target. Secondly, a permittivity map of a slice breast has been obtained, able to localize a malignant tissue inside the adipose region of the breast model. The discussed results represent a useful preliminary assessment for the implementation of a lowcost, compact and non-ionizing breast imaging setup, able to support the currently adopted diagnostic techniques in the framework of breast cancer detection and breast imaging applications.

\section{References}

[1] BELLIZZI, G., BUCCI, O. M., CATAPANO, I. Microwave cancer imaging exploiting magnetic nanoparticles as contrast agent. IEEE Transactions on Biomedical Engineering, 2011, vol. 58, no. 9, p. 2528-2536. DOI: 10.1109/TBME.2011.2158544

[2] COSTANZO, S., LOPEZ, G. Single-step approach to phaseless contrast-source inverse scattering. In Rocha, A., Adeli, H., Reis, L., Costanzo, S. (eds.) New Knowledge in Information Systems and Technologies. WorldCIST'19 2019. Advances in Intelligent Systems and Computing, 2019, vol. 932, p. 278-283. DOI: 10.1007/978-3-030-16187-3_27
[3] VAN DEN BERG, P. M., ABUBAKAR, A. Contrast source inversion method: State of art. Journal of Electromagnetic Waves and Applications, 2001, vol. 15, no. 11, p. 1503-1505. DOI: $10.1163 / 156939301 \times 00067$

[4] VAN DEN BERG, P. M., KLEINMAN, R. E. A contrast source inversion method. Inverse Problems, 1997, vol. 13, no. 6, p. 1607-1620. DOI: $10.1088 / 0266-5611 / 13 / 6 / 013$

[5] NIKOLOVA, N. K. Scalar-wave models in electromagnetic scattering. In Introduction to Microwave Imaging. Cambridge: Cambridge University Press, 2017, p. 1-110. DOI: 10.1017/9781316084267.002

[6] COSTANZO, S., DI MASSA, G., PASTORINO, M., et al. Noninvasive microwave characterization of dielectric scatterers. In Costanzo, S. (ed.) Microwave Materials Characterization. InTech, 2012, p. 38-50. DOI: $10.5772 / 50842$

[7] COLTON, D., KRESS, R. The Helmholtz equation. In Inverse Acoustic and Electromagnetic Scattering Theory, 2013, p. 13-38. DOI: 10.1007/978-1-4614-4942-3_2

[8] BÜRGEL, F., KAZIMIERSKI, K. S., LECHLEITER, A. IPscatt a MATLAB Toolbox for the Inverse Medium Problem in Scattering. 2017.

[9] TSUBURAYA, T., MENG, Z., TAKENAKA, T. Inverse scattering analysis from measurement data of total electric and magnetic fields by means of cylindrical-wave expansion. Electronics, 2019, vol. 8, no. 4, p. 1-11. DOI: 10.3390/electronics 8040417

[10] ZHENG, H., LI, L., LI, F. A multi-frequency MRCSI algorithm with phaseless data. Inverse Problems, 2009, vol. 25, no. 6, p. 1-13. DOI: 10.1088/0266-5611/25/6/065006

[11] LI, L., ZHENG, H., LI, F. Two-dimensional contrast source inversion method with phaseless data: TM case. IEEE Transactions on Geoscience and Remote Sensing, 2009, vol. 47, no. 6, p. 1719-1736. DOI: 10.1109/TGRS.2008.2006360

[12] D’URSO, M., BELKEBIR, K., CROCCO, L., et al. Phaseless imaging with experimental data: Facts and challenges. Journal of the Optical Society of America A, 2008, vol. 25, no. 1, p. 271-281. DOI: $10.1364 /$ JOSAA.25.000271

[13] GEFFrin, J. M., SABOUROUX, P., EYRAUD, C. Free space experimental scattering database continuation: Experimental set-up and measurement precision. Inverse Problems, 2005, vol. 21, no. 6, p. S117-S130. DOI: 10.1088/0266-5611/21/6/S09

[14] BELKEBIR, K., SAILLARD, M. Special section: Testing inversion algorithms against experimental data. Inverse Problems, 2001 , vol. 17 , no. 6, p. 1565-1571. DOI: 10.1088/0266$5611 / 17 / 6 / 301$

[15] COMSOL Multiphysics ${ }^{\circledR}$. v. 5.4. www.comsol.com. COMSOL $\mathrm{AB}$, Stockholm, Sweden

[16] LAZEBNIK, M., POPOVIC, D., MC CARTNEY, L., et al. A large-scale study of the ultrawideband microwave dielectric properties of normal, benign and malignant breast tissues obtained from cancer surgeries. Physics in Medicine and Biology, 2007, vol. 52, no. 20, p. 6093-6115. DOI: 10.1088/0031-9155/52/20/002

\section{About the Authors ...}

Sandra COSTANZO received the Laurea degree (summa cum laude) in Computer Engineering from the University of Calabria in 1996, and the Ph.D. degree in Electronic Engineering from the University of Reggio Calabria in 2000. Currently, she is an Associate Professor at University of Calabria, Italy, where she teaches the courses of 
electromagnetic waves propagation, antennas, remote sensing and radar systems, and electromagnetic diagnostics. At the same University, she is the Coordinator of the Master Degree Course in Telecommunication Engineering. She holds the Italian National Qualification for Full Professor Position in Electromagnetic Fields. Since 1996, she has been involved in many research projects funded by ESA (European Space Agency), ASI (Agenzia Spaziale Italiana), MIUR (Ministero dell'Istruzione, dell'Università e della Ricerca) and private companies. She is Senior Member of IEEE, member of IEEE South Italy Geoscience and Remote Sensing Chapter, CNIT (Consorzio Nazionale Interuniversitario per le Telecomunicazioni) and SIEm (Società Italiana di Elettromagnetismo), and Board Member of IEEEAP/ED/MTT North Italy Chapter, and of IEEE Information Theory Italy Chapter. She is Associate Editor for IEEE Antennas and Wireless Propagation Letters, IEEE Access, IEEE Journal of Electromagnetics, RF and Microwaves in Medicine and Biology, and member of the Edito- rial Board for Radioengineering and International Journal of RF and Microwave Computer-Aided Engineering. Her research interests are focused on near-field far-field techniques, antenna measurement techniques, antenna analysis and synthesis, numerical methods in electromagnetics, millimeter-wave antennas, reflectarrays, microwave sensors for biomedical applications, microwave imaging, electromagnetic characterization of materials, innovative antennas and technologies for radar applications. She has been Editor of 2 books and Lead Editor of 3 special issues on international journals. She has (co)authored more than 170 contributions in international journals, books and conferences.

Giuseppe LOPEZ was born in 1994. He received the Bachelor's Degree in Electronic Engineering in 2016 and the Master's Degree in Telecommunications Engineering in 2019, both from the University of Calabria, Italy. His current research interest is mainly focused on microwave imaging techniques. 